\title{
Portal Vein Thrombosis Associated with Trousseau Syndrome due to Urinary Bladder Squamous Cell Carcinoma in a Liver Cirrhosis Patient
}

\author{
Naruhiro Kimura ${ }^{1}$, Atsunori Tsuchiya ${ }^{1}$, Chiyumi Oda ${ }^{1}$, Atsushi Kimura ${ }^{1}$, Kazunori Hosaka ${ }^{1}$, \\ Kentaro Tominaga ${ }^{1}$, Kazunao Hayashi ${ }^{1}$, Tatsuya Abé ${ }^{2}$, Hajime Umezu ${ }^{3}$ and Shuji Terai ${ }^{1}$
}

\begin{abstract}
:
A 75-year-old woman with liver cirrhosis was admitted for treatment of portal vein thrombosis (PVT). Computed tomography (CT) showed PVT, massive ascites, and multiple abdominal organ embolism. Blood tests revealed a decreased liver function (Child-Pugh grade C). Language impairment followed by progressive left hemi-paralysis was subsequently detected. Magnetic resonance imaging revealed multiple small acute cerebral infarctions and, on CT, a 30-mm bladder tumour; a biopsy specimen examination showed squamous cell carcinoma. Her general condition worsened rapidly, and the best supportive care was chosen. Our findings suggest that, in patients with PVT, Trousseau syndrome should be considered, even in cases of liver cirrhosis.
\end{abstract}

Key words: liver cirrhosis, portal vein thrombosis, squamous cell carcinoma, Trousseau syndrome, small intestine

(Intern Med 59: 1971-1975, 2020)

(DOI: 10.2169/internalmedicine.4112-19)

\section{Introduction}

Trousseau syndrome is known as a malignancy-related thromboembolism that leads to a hypercoagulable state, including arterial or venous thrombosis (1). Professor Armand Trousseau first reported the relationship between cancer and thrombosis in 1865 (2). The clinical presentation of Trousseau syndrome was thought to be mainly deep vein thrombosis (DVT) and pulmonary embolism (PE); however, this syndrome can also cause embolisms in various organs, such as the brain, spleen, and kidney, resulting in a severe condition in patients with malignancies. It is reported to occur in $4-20 \%$ of cancer patients (3), and the risk of venous thrombosis is 4-to-7-fold higher in patients with cancer than in those without cancer (4). However, the prevalence of portal vein thrombosis (PVT) as a clinical consequence of Trousseau syndrome is low.
Conversely, the incidence of PVT is high in patients with advanced liver dysfunction, ranging from $0.6 \%$ to $16 \%$ in patients with well-compensated cirrhosis and approximately $35 \%$ in patients with hepatocellular carcinoma (5). The mechanism underlying PVT in patients with cirrhosis is assumed to involve a decrease in the portal blood flow and levels of natural anticoagulants (5). Liver dysfunction is frequently related to dysregulation of the coagulation balance through decreased levels of protein $\mathrm{C}$ and increased levels of factor VIII (6). However, even in this hypercoagulable state, patients with liver cirrhosis experiencing both PVT and multiple organ embolization are rare.

We herein report a case of PVT caused by Trousseau syndrome in a patient with chronic hepatitis $\mathrm{C}$.

\section{Case Report}

A 75-year-old woman with liver cirrhosis due to hepatitis

\footnotetext{
${ }^{1}$ Division of Gastroenterology and Hepatology, Graduate School of Medical and Dental Sciences, Niigata University, Japan, ${ }^{2}$ Division of Molecular and Diagnostic Pathology, Niigata University Graduate School of Medical and Dental Sciences, Japan and ${ }^{3}$ Division of Pathology, Graduate School of Medical and Dental Sciences, Niigata University, Japan

Received: October 29, 2019; Accepted: February 19, 2020; Advance Publication by J-STAGE: May 23, 2020

Correspondence to Dr. Naruhiro Kimura, nkimura@med.niigata-u.ac.jp
} 

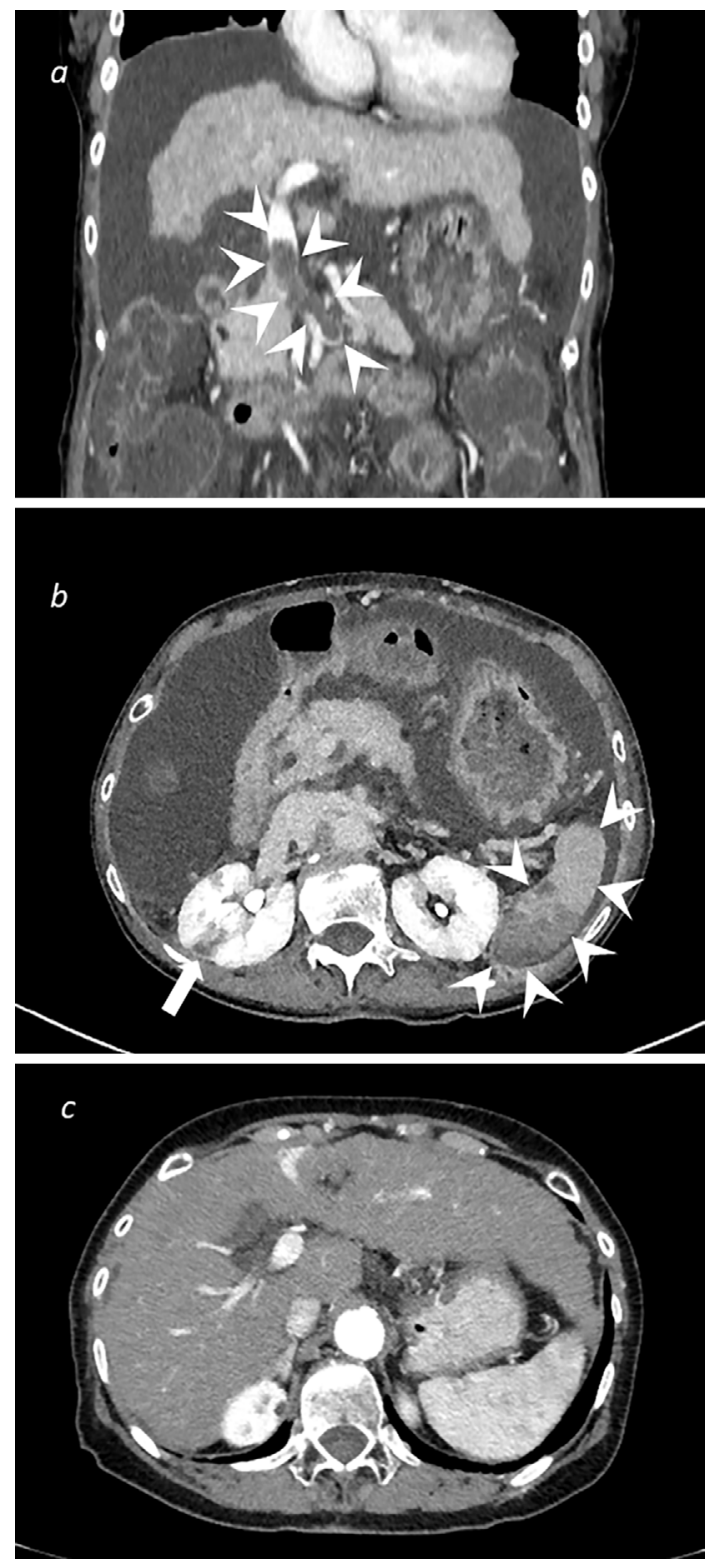

Figure 1. Computed tomography findings. Enhanced computed tomography showed thrombosis from the splenic vein to the portal vein (a; white arrowheads), multiple and broadranging splenic (b; white arrowheads) and multiple small kidney (b; white arrow) infarctions, and massive ascites. A CT image taken one year before hospitalization showed that the patient had liver cirrhosis, but no evidence of portal vein thrombosis nor other organ thromboses (c) was seen.

C was admitted to our hospital for the treatment of PVT and massive ascites detected by ultrasonography and abdominal enhanced-computed tomography (CT). Fifty years prior, she had undergone bladder dilatation using the small intestine due to a neurogenic bladder but had subsequently required intermittent catheterization. Hepatitis $\mathrm{C}$ virus infection was treated using sofosbuvir and ledipasvir, and a sustained virologic response was achieved 10 months before hospitalization when her liver function was classified as Child-Pugh grade A.

One month before admission, the patient felt upper ab- dominal discomfort. On admission, although her abdominal symptoms had improved, abdominal CT revealed liver cirrhosis without hepatocellular carcinoma, a growing thrombosis in the splenic vein to the portal vein (Fig. 1a), multiple and broad-ranging splenic (Fig. 1b) and multiple small kidney (Fig. 1b) infarctions, and massive ascites. Blood tests revealed the rapid reduction in the liver function and an increase in fibrinolytic markers: serum albumin, $2.4 \mathrm{~g} / \mathrm{dL}$ (3.8$5.2 \mathrm{~g} / \mathrm{dL})$; serum total bilirubin, $1.6 \mathrm{mg} / \mathrm{dL}(0.4-1.5 \mathrm{mg} / \mathrm{dL})$;

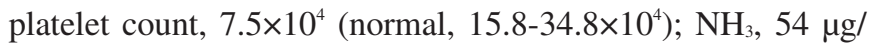
$\mathrm{dL}(12-66 \mu \mathrm{g} / \mathrm{dL})$; prothrombin time, (38\% 70-140\%); PTINR, 1.50 (0.80-1.20); fibrin/fibrinogen degradation products, $44.6 \mu \mathrm{g} / \mathrm{mL}$ (normal, $<5.0 \mu \mathrm{g} / \mathrm{mL}$ ); D-dimer, $27.2 \mathrm{ng} /$ $\mathrm{mL}$ (normal, <1.0 ng/mL); anti-cardiolipin antibody $\mathrm{IgG}$, $1.20 \mathrm{U} / \mathrm{mL}$ (normal, $<10 \mathrm{U} / \mathrm{mL}$ ); anti-cardiolipin antibody $\beta$ 2-GPI, $8.00 \mathrm{U} / \mathrm{mL}$ (normal, $3.5 \mathrm{U} / \mathrm{mL}$ ); and Protein C, $31 \%$ (normal, 82-112\%). Several tumour markers were detected: $\alpha$-fetoprotein, $4 \mathrm{ng} / \mathrm{mL}$ (normal, <19); des- $\gamma$-carboxy prothrombin, $63.9 \mathrm{mAU} / \mathrm{mL}$ (normal, <37.8); carcinoembryonic antigen, $11.2 \mathrm{ng} / \mathrm{mL}$ (normal, <5.0); carbohydrate antigen 19-9, $107 \mathrm{U} / \mathrm{mL}$ (normal, <37.0); carbohydrate antigen 125, $698 \mathrm{U} / \mathrm{mL}$ (normal, <35.0); carcinoembryonic antigen 15-3, $42.1 \mathrm{ng} / \mathrm{mL}$ (normal, <28); and squamous cell carcinoma antigen, $40.9 \mathrm{ng} / \mathrm{mL}$ (normal, <1.5).

Immediately after admission, 14,000 IU/day of heparin was administered to prevent the progression of arterial thrombosis and PVT. However, the day after admission, language impairment followed by progressive left hemiparalysis was detected. Magnetic resonance imaging (MRI) showed multiple small acute cerebral infarctions (Fig. 2). The patient did not have arrhythmia or atrial fibrillation, and echocardiography showed no abnormal findings in her heart. To determine the cause of thrombosis, systemic CT and MRI were performed and revealed two massive urinary stones along with a tumour, $30 \mathrm{~mm}$ in diameter, derived from the bladder in her pelvis (Fig. 3). Cytology of the urine samples and biopsy specimens from the bladder tumour showed squamous cell carcinoma. Since her disease was rapidly progressing, her general condition worsened, and the best supportive care was chosen. However, the patient ultimately died 56 days after admission.

We performed necropsy after obtaining informed consent from her family. Macroscopic pathologic findings showed that the tumour was derived from the small intestine that had been used for bladder dilatation and was infiltrating the posterior bladder wall and bladder cavity (Fig. 4a). Histological findings confirmed squamous cell carcinoma (Fig. 4b) in the transplanted small intestine that had invaded and expanded to her original bladder and bladder cavity (Fig. 4c, d). No metastasis was detected. Based on these findings, we suspected Trousseau syndrome due to the squamous cell carcinoma derived from the urinary bladder.

\section{Discussion}

We herein report a case of PVT caused by Trousseau syn- 

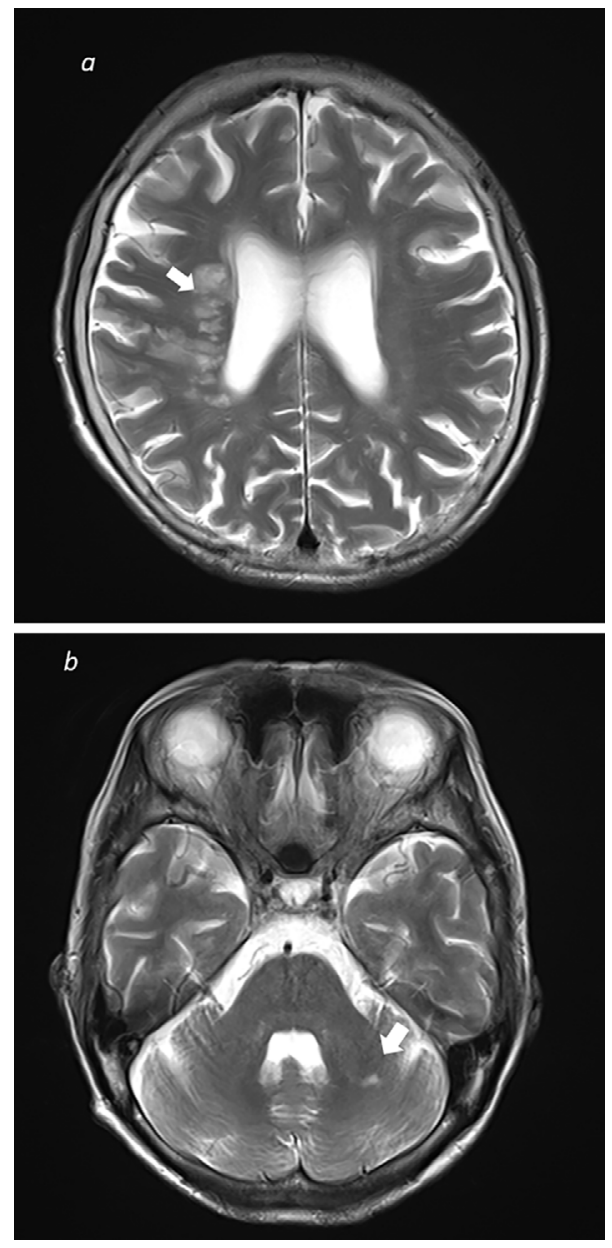

Figure 2. Magnetic resonance imaging findings. Magnetic resonance imaging showed multiple small acute cerebral infarctions (a, b; white arrows).

drome due to urinary bladder carcinoma in a liver cirrhosis patient. The first clinical thrombosis in this case was detected in the patient's portal vein, which although common in liver cirrhosis patients, is rarely seen in Trousseau syndrome. We had originally speculated that the PVT was due to the decompensated state of liver cirrhosis; however, many of the thrombosis-related symptoms, including cerebral infiltration and multiple organ embolization, occurred after admission. Given that liver cirrhosis has a strong impact on both PVT and Trousseau syndrome, we suspect that Trousseau syndrome can be complicated with PVT under certain conditions when a low serum albumin level secondary to liver cirrhosis exists.

The first clinical symptom of Trousseau syndrome tends to be DVT and/or artery thrombosis, but PVT as the first clinical manifestation is rare. Venous thromboembolism (VTE) is a common clinical condition and includes DVT and PE. A recent study showed that the prevalence of VTE is $4-20 \%$ among cancer patients (3). Furthermore, arterial thrombosis reportedly occurs in $1.5-3.1 \%$ of cancer patients and is especially prevalent among patients with pancreatic and gynaecological tumours (7). These thrombosis events, as part of Trousseau syndrome, are thought to be related to the
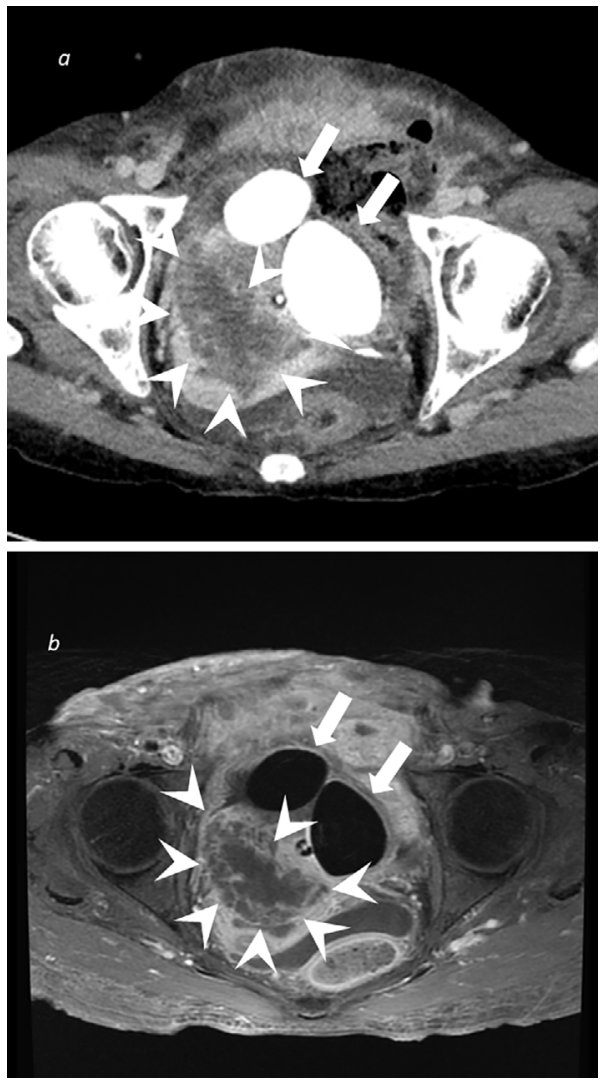

Figure 3. Computed tomography and magnetic resonance imaging. Enhanced-computed tomography and magnetic resonance imaging showed two urinary stones (a, b, white arrows) and a tumour 30-mm in diameter in the pelvis derived from the bladder (a, b, white arrowheads).

increased secretion of tissue factors from solid tumours. Elevated tissue factor levels are postulated to promote hypercoagulability, tumour aggressiveness, and angiogenesis $(8,9)$. In addition to a hypercoagulable state, liver dysfunction is also associated with a disrupted coagulation function (6). A recent study showed that serum albumin could act as a modulator of platelet activation in liver cirrhosis patients and that a low serum albumin level was associated with PVT (10). Our patient had all of these factors-a solid tumour in the bladder, liver cirrhosis, and a reduced serum albumin level $(2.4 \mathrm{~g} / \mathrm{dL})$-which cumulatively may have contributed to PVT.

Trousseau syndrome is cancer-related and has been thought to have a varied prevalence depending on the cancer-bearing organ. Trousseau syndrome has been reported to occur in patients with carcinomas of the lung (17\%), pancreas $(10 \%)$, colon and rectum $(8 \%)$, kidney $(8 \%)$, and prostate $(7 \%)(11)$. To our knowledge, however, there are only three reports describing cases wherein Trousseau syndrome was caused by urinary bladder cancer (12-14). In one of these case reports, a 38-year-old woman had a neurogenic bladder requiring intermittent catheterization for 30 years, as in our case. Although squamous cell carcinoma of the urinary bladder comprises only $2.4 \%$ of all urinary bladder cancers (15), chronic inflammation, such as infection of 


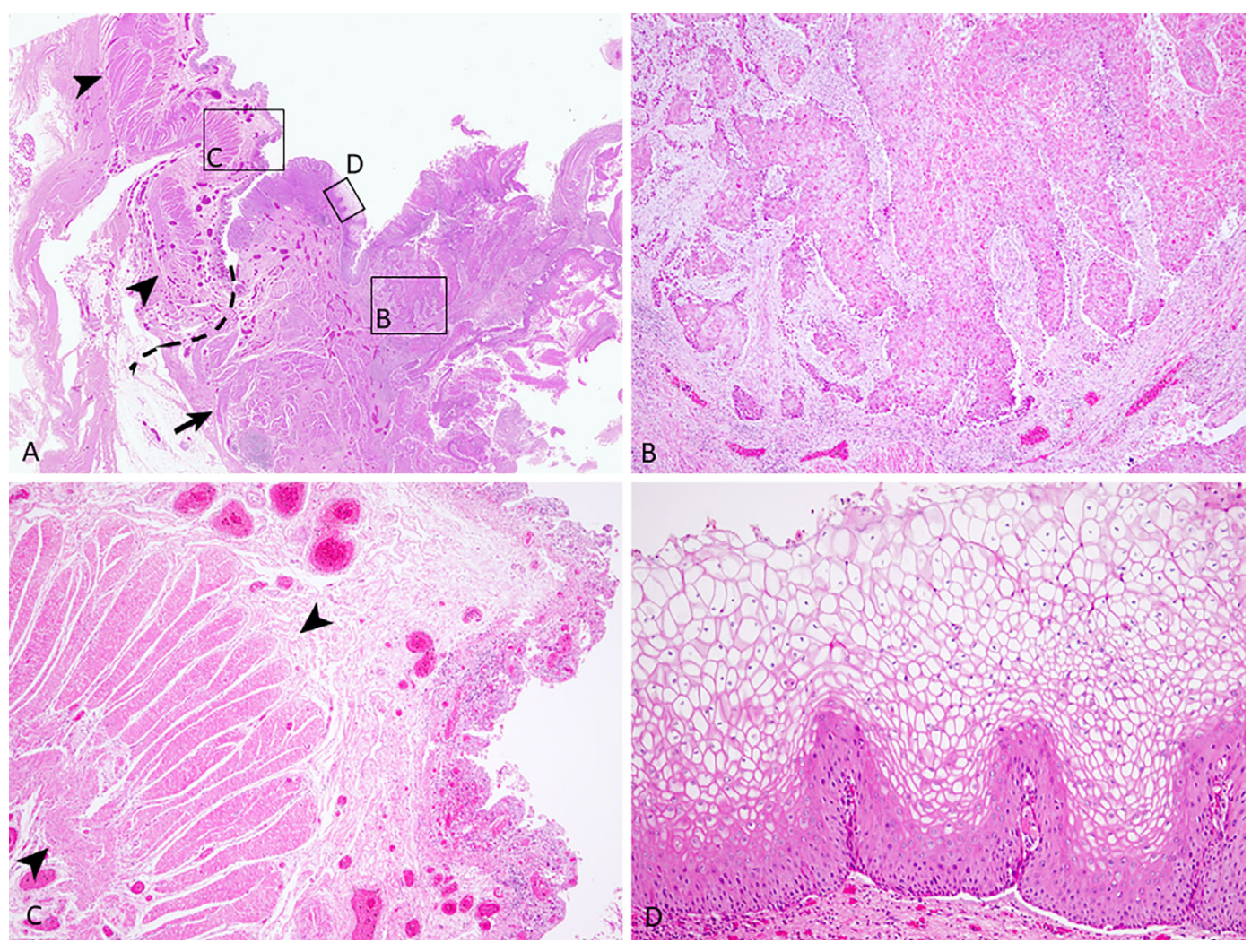

Figure 4. Histological findings of squamous cell carcinoma arising from the urinary bladder. (a) Low-magnification image of the borderline area between the urinary bladder and the transplanted small intestine. (b) Squamous cell carcinoma of the urinary bladder. (c) The transplanted small intestine. (d) Metaplastic squamous cell epithelium of the bladder. Hematoxylin and Eosin staining (a-d). Original magnifications: $\times \mathbf{5}(\mathrm{a}), \times \mathbf{4 0}(\mathrm{b}, \mathrm{c})$, and $\times \mathbf{1 0 0}(\mathrm{d})$. Arrow: muscularis propria of the bladder. Arrowheads: muscularis propria of the transplanted intestine.

Schistosoma haematobium, results in squamous metaplasia of the transitional epithelium (16). We therefore speculated that chronic inflammation due to a neurogenic bladder, catheter invasion, and bladder stone might have triggered oncogenesis in the bladder of both patients. Furthermore, we assumed that, due to this transformation of squamous metaplasia, our patient had an increased number of tumour markers, such as the squamous cell carcinoma marker SCC antigen and other adenocarcinoma markers, including carcinoembryonic antigen (CEA), CA19-9, and CA15-5. The increased protein induced by vitamin $\mathrm{K}$ absence of antagonist (PIVKA) II value may have been due to the reduction in the vitamin $\mathrm{K}$ level by liver cirrhosis.

Both the location and histological type of carcinoma affect the incidence of embolisms. Furthermore, squamous cell carcinoma is rarely associated with Trousseau syndrome, while adenocarcinoma has a 1.65 -fold higher probability of inducing an embolism than squamous carcinoma (17). Our case report is also novel in this regard.

In conclusion, the findings of this case suggest that when patients present with PVT, Trousseau syndrome should be considered as a diagnosis, even in cases of liver cirrhosis.

The authors state that they have no Conflict of Interest (COI).

\section{References}

1. Varki A. Trousseau's syndrome: multiple definitions and multiple mechanisms. Blood 110: 1723-1729, 2007.

2. Trousseau A. Phlegmasia alba dolens. Clin Med Hotel-dieu Paris 3: $654-712,1865$.

3. Khorana AA, Francis C, Culakova E, Kuderer NM, Lyman GH. Thromboembolism is a leading cause of death in cancer patients receiving outpatient chemotherapy. Int Soc Thromb Haemost 5: 632-634, 2005

4. Stein PD, Beemath A, Meyers FA, Skaf E, Sanchez J, Olson RE. Incidence of venous thromboembolism in patients hospitalized with cancer. Am J Med 119: 60-68, 2006.

5. Sameer P, Riddhi S, Prashant K. Portal vein thrombosis. Am J Med 123: 111-119, 2010.

6. Intagliata NM, Caldwell SH, Tripodi A. Diagnosis, development, and treatment of portal vein thrombosis in patients with and without cirrhosis. Gastroenterology 156: 1582-1599.e1, 2019.

7. Sanon S, Lenihan DJ, Mouhayar E. Peripheral arterial ischemic events in cancer patients. Vasc Med 16: 119-130, 2011.

8. Rickles FR. Mechanisms of cancer-induced thrombosis in cancer. Pathophysiol Haemost Thromb 35: 103-110, 2006.

9. Rak J, Yu JL, Luyendyk J, Mackman N. Oncogenes, trousseau syndrome, and cancer-related changes in the coagulome of mice and humans. Cancer Res 66: 10643-10646, 2006.

10. Basili S, Carnevale R, Nocella C, et al. Serum albumin is inversely associated with portal vein thrombosis in cirrhosis. Hepatol Commun 3: 504-512, 2019.

11. Sørensen HT, Mellemkjaer L, Olsen JH, Baron JA. Prognosis of 
cancers associated with venous thromboembolism. N Engl J Med 343: 1846-1850, 2000.

12. Kato T, Yasuda K, Iida H, et al. Trousseau's syndrome caused by bladder cancer producing granulocyte colony-stimulating factor and parathyroid hormone-related protein: a case report. Oncol Lett 12: 4214-4218, 2016

13. Kayukawa S, Ina K, Furuta R, et al. Bladder squamous cell cancer accompanied by Trousseau's syndrome: a case report. Clin Case Rep 6: 653-657, 2018.

14. Orrell RW, James-Galton M, Stevens JM, Rossor MN. Cerebral achromatopsia as a presentation of Trousseau's syndrome. Postgrad Med J 71: 44-46, 1995.

15. Royce TJ, Lin CC, Gray PJ, Shipley WU, Jemal A, Efstathiou JA.
Clinical characteristics and outcomes of nonurothelial cell carcinoma of the bladder: results from the National Cancer Data Base. Urol Oncol 36: 78.e1212, 2018.

16. Dematei A, Fernandes R, Soares R, Alves H, Richter J, Botelho MC. Angiogenesis in Schistosoma haematobium-associated urinary bladder cancer. APMIS 12: 1056-1062, 2017.

17. Bick RL. Coagulation abnormalities in malignancy: a review. Semin Thromb Hemost 18: 353-372, 1992.

The Internal Medicine is an Open Access journal distributed under the Creative Commons Attribution-NonCommercial-NoDerivatives 4.0 International License. To view the details of this license, please visit (https://creativecommons.org/licenses/ by-nc-nd/4.0/).

(C) 2020 The Japanese Society of Internal Medicine Intern Med 59: 1971-1975, 2020 